\title{
L'empire du réseau parallèle : le numérique en chine
}

The empire of the parallel network: China's digital platform

El imperio de la red paralela: el ámbito digital en China

\section{Ghislaine Azémard et Matthieu Quiniou}

\section{OpenEdition}

\section{Journals}

Édition électronique

URL : http://journals.openedition.org/ctd/327

DOI : $10.4000 /$ ctd. 327

ISSN : 2491-1437

Éditeur

Chaire Unesco Pratiques émergentes en technologies et communication pour le développement

Référence électronique

Ghislaine Azémard et Matthieu Quiniou, "L'empire du réseau parallèle : le numérique en chine », Communication, technologies et développement [En ligne], 5 | 2018, mis en ligne le 02 janvier 2018, consulté le 21 avril 2019. URL : http://journals.openedition.org/ctd/327 ; DOI : 10.4000/ctd.327

Ce document a été généré automatiquement le 21 avril 2019

Communication, technologies et développement 


\title{
L'empire du réseau parallèle : le numérique en chine
}

\author{
The empire of the parallel network: China's digital platform \\ El imperio de la red paralela: el ámbito digital en China
}

Ghislaine Azémard et Matthieu Quiniou

\section{NOTE DE L'AUTEUR}

\section{INDONÉSIEN}

Abstrakt: Zwischen der Suche nach Autarkie bei digitalen Diensten und der Stärkung der Position der chinesischen Hardwareindustrie auf dem Weltmarkt ist der chinesische Staat nicht bereit, sich in eine soziale und kulturelle Globalisierung einzubringen, sondern will die Vorteile der kommerziellen Globalisierung voll ausschöpfen

1 La Chine entre difficilement dans la catégorie Nord ou Sud, ou de pays « développé » ou « en voie de développement », en raison de sa vitesse de développement, de sa puissance économique globale et du niveau de vie moyen de ses ressortissants. Elle s'analyse non pas par rapport aux pays développés, mais par rapport à l'Occident, c'est-à-dire selon un rapport est-ouest, où la différence est plus d'ordre culturel que purement économique. La classification de BRICS (Brésil, Russie, Inde, Chine et Afrique du Sud) semble avoir moins de pertinence aujourd'hui, du moins sous l'angle économique. Un nouveau concept, celui des TICKS, apparaît notamment dans les milieux financiers pour élaborer des fonds d'investissement ${ }^{1}$ et intègre la Chine dans une catégorie plus homogène aux côtés de Taïwan, de l'Inde et de la Corée du Sud. La Chine est actuellement présentée comme une alternative aux États-Unis, comme une nouvelle polarité, une Asie en développement, qui déplace fondamentalement le centre de gravité commercial et impose un questionnement structurel à l'ethnocentrisme occidental.

2 Si le rattrapage technologique de la Chine dans le numérique a été fulgurant, il s'est fait en maintenant et en affirmant une indépendance absolument inédite par rapport aux 
États-Unis et à ses entreprises. Entre recherche d'autarcie pour les services numériques et renforcement de la place des industries chinoises du hardware dans le marché mondial, l'État chinois n'est pas prêt à se fondre dans une mondialisation sociale et culturelle, mais souhaite profiter pleinement de la mondialisation commerciale.

\section{Une politique chinoise d'Internet}

\section{La gouvernance d'Internet bousculée par l'expression de la souveraineté chinoise}

3 La politique chinoise par rapport à Internet diverge profondément de l'idéologie californienne du Web. La quête d'une neutralité absolue du Net n'est pas, pour l'instant, la préoccupation première pour les usagers et activistes chinois.

4 La Chine a cherché à s'émanciper de l'ICANN en pratiquant notamment la politique de la chaise vide aux réunions multi-parties prenantes ${ }^{2}$. Le gouvernement chinois a aussi indiqué à plusieurs reprises qu'il envisageait l'élaboration de racines indépendantes, en arguant principalement de la lenteur de l'ICANN à intégrer les sinogrammes dans les noms de domaine de premier niveau. Cette menace de création d'un Internet parallèle n'a pas été mise à exécution et n'est plus évoquée.

5 Si la Chine n'est pas indépendante de la gouvernance de l'ICANN, le projet Bouclier d'or, aussi appelé Great Firewall par analogie avec la muraille de Chine, existait déjà depuis 2003 avec, pour conséquence, une étanchéité importante entre l'«Internet chinois " et l'«Internet global». Initialement occultée par le Gouvernement, la question du contournement depuis la Chine du système de blocage des sites est devenue un enjeu important. Si ces contournements du système de blocage bénéficiaient d'une certaine tolérance, cette situation s'expliquait plus par le fait que ces pratiques étaient issues d'étrangers dans le cadre de leurs usages non professionnels que par un manque de moyens ou des difficultés techniques. Le renforcement des méthodes visant à lutter contre le contournement de la muraille numérique s'explique par la crainte d'une perte de contrôle de la diffusion de l'information par la généralisation à l'ensemble de la population chinoise des pratiques des expatriés en Chine et de la diaspora réimplantées. La campagne généralisée du gouvernement chinois contre Tor et les réseaux privés virtuels (VPN) date approximativement de 2010, et le premier cas recensé de dysfonctionnement de Tor en Chine remonte au 4 octobre 20113. Depuis 2015, les principaux fournisseurs de VPN ont des difficultés à offrir un service stable ${ }^{4}$ et les principales failles de la muraille numérique de Chine ont été résorbées.

6 La souveraineté de l'État sur l'Internet chinois s'étend aussi aux services accessibles depuis la Chine, avec la mise à l'écart de nombreux acteurs étrangers, à l'image de Google ou de Facebook.

\section{L'alternative chinoise aux GAFA : entre réplication des systèmes et différence d'usages}

7 Des premiers CD-Rom aux dernières applications mobiles, les contenus numériques chinois ont toujours été marqués par un style propre. S'il ne peut être contesté que les grands sites américains, hégémoniques en Occident, ont influencé les sites chinois du 
même secteur dès le stade de leur création, il convient cependant de noter que ces services ont été profondément réadaptés pour la société et la culture chinoises.

Si nous prenons l'exemple des usages actuels du téléphone mobile en Chine, nous pouvons constater également dans ce secteur certaines spécificités à l'œuvre. Les internautes chinois utilisent le téléphone mobile: avec 500 millions de Smartphones vendus en 2015, trois fois plus qu'aux États- Unis, la Chine représenterait un tiers du marché mondial $5.85 \%$ des Chinois se tourneraient vers des entreprises nationales (Lenovo, Huawei et ZTE) pour acquérir un mobile. Cet engouement est en partie motivé par les nouveaux usages qu'il autorise, en particulier la consultation d'Internet et l'ensemble des interactions que cela entraîne. Suivant les chiffres du "Statistical Report on Internet Development » du CNIC, on est passé de $24 \%$ d'internautes chinois utilisant le téléphone mobile pour aller sur Internet en 2007 à $74,5 \%$ en $2012^{6}$. Les internautes chinois sont connectés environ trois heures par jour, mais plus de la moitié de ce temps sur mobile. Ils surfent et regardent des vidéos en ligne. On constate l'abandon de l'écran de télévision pour le mobile.

Les usages chinois se distinguent sensiblement de ceux des Occidentaux sur des points parfois inattendus. C'est le cas par exemple des fonctionnalités liées aux messages vocaux, qui figurent parmi les incontournables en Chine alors qu'elles peinent à gagner en visibilité en Occident, malgré la recherche de synergie entre les acteurs, comme entre Facebook et Skype. À ce titre, il est intéressant de noter que la messagerie instantanée de Microsoft, MSN Messenger, était encore largement utilisée en Chine, notamment pour les visioconférences, alors qu'elle était déjà abandonnée en Occident. MSN Messenger n'a d'ailleurs été complètement abandonné en Chine qu'en 2014, alors qu'il avait déjà fermé un an auparavant dans le reste du monde. MSN Messenger a été concurrencé et progressivement remplacé dans les usages chinois par les applications du groupe Tencent Holdings : QQ et WeChat. Souvent considérées comme des copies serviles d'ICQ pour QQ et de WhatsApp pour WeChat, ces applications chinoises ont pu s'imposer parce que les usages chinois, sans être uniformes, sont différents de ceux de l'Occident.

Il convient de pondérer l'allégation de copie régulièrement faite aux plateformes chinoises. Même si ces dernières ont rarement été les premières à ouvrir un champ sectoriel et à innover en design d'interface, c'est aussi le cas des géants du Net, comme Google ou de Facebook, qui ont également été précédés d'autres plateformes dans leur secteur. Ce qui a permis à Google de s'imposer en Occident est principalement la pertinence de son algorithme rompant avec une approche purement sémantique. Pour Facebook, le succès est lié en grand partie à l'obligation faite aux utilisateurs de s'identifier avec leur patronyme et à un modèle de dissémination particulièrement recherché invitant les utilisateurs à envoyer des invitations indirectes à leurs contacts mail sous prétexte de leur permettre d'être identifiés sur des photographies communes mises en ligne sur le réseau. Pour expliquer la différence de succès entre WhatsApp et WeChat, il convient de s'intéresser au public visé et à ses habitudes. WhatsApp était principalement utilisé en Occident dans les pays n'ayant pas ajusté les prix des SMS aux possibilités des Smartphones, et s'est maintenu pour les communications instantanées mobiles vers l'étranger encore parfois surtaxées. À l'inverse, la majorité des usagers chinois préfèrent ce type de communication instantanée multifonctionnelle à la rigidité des SMS, ce qui explique le succès de WeChat, application quasi exclusivement destinée à un public chinois pour l'instant. 
11 La réussite en Chine des sites et applications développés par des entreprises chinoises s'explique aussi par les obstacles juridiques et politiques, notamment en termes de droit des investissements en Chine dans les secteurs des médias, ou encore de censure. Il est intéressant de remarquer que le modèle chinois de réplication et de défense sectorielle se développe dans d'autres pays dans les derniers secteurs transformés par le numérique. C'est le cas par exemple pour l'application Uber, notamment en France, où elle est, d'une part, partiellement prohibée suite à un sursaut corporatiste des taxis et des pouvoirs publics et, d'autre part, concurrencée par des applications françaises adaptées aux habitudes de consommation françaises du transport privatif, comme Heetch, ne fonctionnant que dans les grandes villes et en soirée.

\section{Le numérique en Chine : un secteur qui ne connaît pas la crise}

12 La croissance économique de la Chine fait l'objet de nombreuses inquiétudes, mais les scénarios, même les plus alarmistes, considèrent qu'un secteur qui sera épargné par celleci et dont le développement devrait être accéléré est celui du numérique et des nouvelles technologies. Il devrait croître de manière importante dans un marché domestique national imposant, avec des marges de progression inégalée dans le monde. Les dépenses en matière de nouvelles technologies de l'information et de la communication devraient dépasser les 475 millions de dollars, enregistrer un taux de croissance de $11 \%$ et atteindre $43 \%$ de la croissance mondiale du secteur technologique. Les objectifs gouvernementaux sont affichés : les industries nouvelles devraient atteindre très rapidement $8 \%$ du produit intérieur brut, et la connexion de tous les Chinois est prévue à très court terme. 700 millions seraient déjà connectés, soit près de la moitié de la population et plus de $20 \%$ des usagers mondiaux d'Internet ${ }^{7}$.

13 Ce secteur en pleine expansion apparaît comme un objectif majeur de la Chine dans sa stratégie de rattrapage technologique mondial et dans la pénétration de son modèle de développement, en particulier en direction des pays africains.

\section{Le numérique en Chine}

\section{Les privilégiés du numérique...}

14 La propagation d'Internet et de ses usages reste encore très inégalitaire en Chine. Les grandes métropoles et les zones côtières bénéficient de services que la Chine rurale ne peut encore espérer. Le pourcentage d'internautes varie du simple au double en fonction des régions, en raison d'un déploiement des infrastructures de télécommunication géographiquement très inégal.

15 Contrairement à un type de pénétration homogène entre les tranches d'âge qui s'est opéré progressivement en France ces dernières années, où l'on constate un véritable nivellement générationnel, en Chine, ce sont les jeunes actifs qui constituent le cœur de cible des usages et la plus forte catégorie de population connectée. $81 \%$ des internautes ont moins de 40 ans $^{8}$. Les jeunes enfants et les personnes âgées ne sont pas des consommateurs de services numériques contrairement à la France où, des très jeunes enfants aux séniors, des tablettes aux objets connectés, les outils et usages numériques traversent les générations et les catégories sociales. 


\section{De l'assemblage à l'innovation : la longue marche du numérique chinois}

La réactivité des entreprises et des créateurs d'entreprises par rapport aux mutations socioéconomiques liées au numérique n'est plus l'apanage de la Californie. En Chine, des pôles de compétitivité colossaux ont été développés, comme à Pékin, avec le quartier Zhongguancun, ou à Shenzhen. À la différence d'un modèle présenté comme auto-généré par les entreprises comme dans la Silicon Valley, le modèle chinois de regroupement d'entreprises et de mise en réseau est planifié. Cette planification n'est pas limitée à la mise en place d'incubateurs, d'infrastructures spécifiques ou à quelques avantages fiscaux mineurs en fonction de la localisation du siège social. Créée à l'initiative du père de Xi Jinping, Xi Zhongxun, avec le soutien de Deng Xiaoping dès la fin des années 1970, pour expérimenter le capitalisme et l'ouverture aux investissements étrangers ${ }^{9}$, la ville de Shenzhen est devenue le siège de la majorité des grandes entreprises high-tech (Tencent, Huawei...). Elle bénéficie, en tant que zone économique spéciale, d'un régime juridique particulier, notamment en matière d'investissement, d'imposition, d'accès au crédit, de mode de règlement des différends et de commerce international.

Ces pôles de compétitivité sont aussi des zones de recherche et de développement s'inscrivant dans la politique du gouvernement chinois qui a pour objectif de faire de la Chine une grande puissance technologique d'ici à $2020^{10}$. Les dépôts de brevets par des résidents chinois ont ainsi été multipliés par plus de dix en dix ans ${ }^{11}$ et les exportations chinoises de produits de haute technologie ont été multipliées par 100 en 20 ans $^{12}$. Cependant, il doit être noté que les brevets déposés par des résidents chinois en Chine sont souvent considérés en moyenne comme se rapportant à des inventions d'une « qualité » moindre ou d'un degré d'innovation inférieur aux inventions déposées par des sociétés étrangères. Ce constat a d'ailleurs été confirmé en grande partie par des études menées par des chercheurs de l'Académie des sciences de Chine ${ }^{13}$.

Concernant l'export de produits de haute technologie, les chiffres doivent aussi être pondérés ; d'une part, ils s'expliquent quasi exclusivement par la place centrale que joue la Chine dans l'assemblage de pièces électroniques, ce qui représente une création de valeur ajoutée limitée en fin de chaîne de production et, d'autre part, de nombreuses entreprises d'assemblage implantées en Chine ne sont pas chinoises. Par ailleurs, certaines entreprises ayant leur siège à Taipei jouent un rôle central en Chine, comme Foxconn, qui est la principale société exportatrice depuis la Chine, tous secteurs confondus, et qui est notamment la sous-traitante d'Apple pour les iPhones.

Encore assimilée il y a quelques années à l'atelier du monde, la Chine reste un lieu d'assemblage de produits dont la valeur ajoutée provient de droits de propriété intellectuelle. Outre la contrefaçon organisée, il y existe une culture populaire de la contrefaçon ${ }^{14}$. Deux mots désignent en Chine la contrefaçon: le terme jiazào $0^{15}$, pour désigner la contrefaçon de manière générique, et le terme shān zhài ${ }^{16}$, qui désigne la culture de la contrefaçon artisanale bas de gamme, en marge des circuits officiels. Le domaine qui nous intéresse, le secteur de l'électronique, et particulièrement celui des Smartphones ${ }^{17}$, est marqué par un niveau élevé de contrefaçon. Ces produits contrefaisants se retrouvent dans les marchés thématiques des gratte-ciel des grandes villes, ou même dans de faux magasins de marques renommées, comme les faux magasins Apple découverts par hasard par des expatriés américains à Kumming en $2011^{18}$. 
20 Si le développement du numérique en Chine a été accompagné par de nombreux scandales quant aux conditions de travail des ouvriers dans le secteur électronique, si le respect des droits de la propriété intellectuelle reste problématique pour une économie globalisée et si l'Internet chinois ne répond pas aux standards de liberté d'expression occidentaux, le rattrapage sociotechnique opéré en quelques années sur la majorité du territoire chinois ne peut être qu'unanimement constaté. Le modèle numérique de la Chine apparaît comme une alternative solide au modèle américain, comme la version numérique de l'économie socialiste de marché.

\section{Le modèle numérique chinois et l'Afrique}

21 La Chine se positionne de manière très originale dans sa relation avec le continent africain. Dans un premier temps, les entreprises chinoises ont apporté en Afrique des outils technologiques à des prix très compétitifs. À ce titre, Huawei, constructeur électronique chinois installé à Shenzhen, s'est implanté en Afrique depuis 1999. Cette entreprise s'est associée à Microsoft en 2013 pour conquérir le marché africain du Smartphone ${ }^{19}$ et a depuis dépassé l'entreprise californienne en Afrique dans ce secteur ${ }^{20}$. Aujourd'hui, Huawei ambitionne de vendre des téléphones haut de gamme à une classe moyenne africaine en demande de services enrichis. La société chinoise déploie aussi les infrastructures de télécommunication sur le continent africain, notamment la fibre optique et la $4 \mathrm{G}^{21}$.

La réussite des entreprises chinoises sur son marché interne et sur le marché africain stimule entreprisesafricainesetprésenteunmodèlealternatifentrepreneurialpourlesnouvellesgénérations en Afrique. Le modèle chinois de l'industrie numérique y a déjà une influence importante, notamment pour la production de téléphones portables low cost. L'entreprise chinoise spécialisée dans les téléphones intelligents Xiaomi, basée à Pékin, est l'une des sociétés à la croissance la plus fulgurante de l'histoire. Xiaomi a déjà inspiré certaines entreprises africaines, comme l'entreprise Tecno, dans le secteur de la téléphonie mobile, au Nigeria ${ }^{22}$

À l'inverse d'un iPhone ou d'un objet communicant en édition limitée qui affiche sa marque comme une distinction sociale, la logique portée par ces entreprises chinoises a l'ambition de toucher la population le plus largement possible. L'approche chinoise des technologies est paradoxalement plus proche de l'ensemble de la population africaine et peut plus facilement être adaptée sur le continent qu'une approche occidentale, qui ne massifie les produits technologiques qu'après appropriation préalable par l'élite.

Les entreprises chinoises aident, par ailleurs, la création de start-up africaines pour définir des applications adaptées au public africain, sur un modèle proche de celui qui a fait le succès des applications chinoises inspirées d'applications occidentales.

\section{Conclusion}

La Chine a réussi à construire sur son vaste marché intérieur un modèle de développement original qui, après avoir généré ses géants nationaux, s'exporte, en particulier en Afrique, où son modèle devient une source d'inspiration pour les entreprises émergentes de ces pays en voie de développement. 


\section{BIBLIOGRAPHIE}

Angelson Jessica, « Are You Listening, Steve Jobs? », This Woman's Work. Notes From a New York City Midwife, 2011, disponible sur https://birdabroad.wordpress.com.

Arsène Séverine, «Chine : Internet, levier de puissance nationale ", Politique étrangère, $\mathrm{n}^{\circ}$ 2012/2 (Internet : outil de puissance), 2012, p. 291-303, disponible sur https://www.cairn.info Banque mondiale, «Exportations de haute technologie (\$ US courants) », disponible sur https:// digitalcommons.law.umaryland.edu.

Campbell Patricia E., Pecht Michael, « The Emperor's New Clothes : Intellectual Property Protections in China ", Journal of Business \& Technology Law, vol. 7, no1, 2012, p. 69-115, disponible sur https://digitalcommons.law.umaryland.edu.

Carsten Paul, Rajagopalan Megha, Wee Sui-Lee, «In China, VPN Internet Access Tools Suffer Further Disruptions », Reuters, 2015, disponible sur https://www.reuters.com.

Chen Shin-Horng, Wen Pei-Chang, «The Evolution of China's Mobile Phone Industry and GoodEnough Innovation », Chung-Hua Institution for Economic Research, Taiwan, 2013, disponible sur http://www.cier.edu.tw.

Faramawy Ali, « Prioritising Africa - Introducing Microsoft 4Afrika », Microsoft TechNet 2013, disponible sur https://blogs.technet.microsoft.com.

Johnson Steve, « The Brics Are Dead. Long Live the Ticks : Tech-Heavy Taiwan, India, China and Korea Are the New Darlings of the EM World », Financial Times2016, disponible sur https:// www.ft.com.

Kuhn Robert Lawrence, How China's Leaders Think: The Inside Story of China's Past, Current and Future Leaders, John Wiley \& Sons, Singapour/Hoboken, 2011.

Le Belzic Sébastien, « Hong Kong : un modèle pour les start-up africaines », Le Monde Afrique, 2015, disponible sur https://www.lemonde.fr.

Le Belzic Sébastien, « Huawei : la marque chinoise de télécoms qui monte sur le continent africain », Le Monde Afrique, 2015 (mis à jour en 2016), disponible sur https://www.lemonde.fr.

Organisation mondiale de la propriété intellectuelle, «Statistiques de propriété intellec- tuelle par pays. Chine », Organisation mondiale de la propriété intellectuelle, Genève, 2015, disponible sur http://www.wipo.int/portal/en/index.html.

Quality Brands Protection Committee, « Chinese Shanzhai Handset Market Research Report 2013 ", rapport, China Association of Enterprises With Foreign Investment, Pékin, 2013, disponible sur http://www.qbpc.org.cn.

Quiniou Matthieu, « Le contentieux du transfert de connaissances dans les relations entre l'Union européenne et la Chine », thèse de doctorat, université Paris II - Panthéon-Assas, Paris, 2015.

Song Hefa, Li Zhenxing, " Patent Quality and the Measuring Indicator System : Comparison Among China Provinces and Key Countries ", papier rédigé à l'occasion de la XIVe Intellec- tual Property Scholars Conference (Berkeley, 7-8 août 2014), 2014, disponible sur https:// www.law.berkeley.edu. 
Song Hefa, Mu Rongping, Chen Fang, Li Zhenxing, « Patent Quality Indicator System : Measurement Based on China Invention Patent Data », Sciences Research Management, $\mathrm{n}^{\circ} 11,2014$, p. 68-76.

Winter Philipp, Lindskog Stefan, « How the Great Firewall of China is Blocking Tor », papier rédigé à l'occasion du IIe USENIX Workshop on Free and Open Communications on the Internet (Bellevue, 6 août 2012), 2012, disponible sur https://www.usenix.org.

\section{NOTES}

1. Johnson, 2016.

2. Voir sur ce point Arsène, 2012.

3. https://trac.torproject.org/projects/tor/ticket/4185; voir aussi sur ce point et plus généralement Winter, Lindskog, 2012.

4. Voir par exemple Carsten, Rajagopalan, Wee, 2015.

5. International Data Corporation Technology et CNIC.

6. Ibidem

7. International Data Corporation Technology et CNIC.

8. Ibidem

9. Pour une présentation de la création de Shenzhen, et plus généralement des symboles politiques en Chine, voir Kuhn, 2011.

10. Conseil des affaires de l'État de la République de Chine, «Plan de développement scientifique de moyen et long termes (2006-2020)» [国家中长期科学和技术发展规划纲要 (2006-2020年)], 31 janvier 2006.

11. Organisation mondiale de la propriété intellectuelle, 2015.

12. Cette comparaison a été obtenue à partir des chiffres publiés par la Banque mondiale : la valeur (en milliard de dollars américains) des exportations de produits de haute technologie était en Chine de 5,247 en 1993 et de 560,058 en 2013; en France, elle était de 30,538 en 1993 et de 108,364 en 2013 (Banque mondiale).

13. Song, Li, 2014 ; Song, Mu, Chen, Li, 2014.

14. Voir sur ce point Quiniou, 2015.

15. 假造, c'est-à-dire contrefaçon.

16. 山寨, c'est-à-dire imitation.

17. Voir notamment Quality Brands Protection Committee, 2013 et Chen, Wen, 2013.

18. Angelson, 2011. Sur cette affaire, voir aussi, sous un angle juridique, Campbell, 2012.

19. Faramawy, 2013.

20. Le Belzic, 2015 (mis à jour en 2016).

21. Ibidem

22. Le Belzic, 2015.

\section{RÉSUMÉS}

Entre recherche d'autarcie pour les services numériques et renforcement de la place des industries chinoises du hardware dans le marché mondial, l'État chinois n'est pas prêt à se fondre 
dans une mondialisation sociale et culturelle, mais souhaite profiter à plein de la mondialisation commerciale.

In search of self-sufficiency for digital services and strengthening of the position of chinese hardware industries in the global market, the chinese state is not ready to merge in a social and cultural globalization, but wants to take full advantage of the commercial globalization.

INDEX

Keywords : China, globalization, industry, trade, digital

Mots-clés : Chine, mondialisation, industrie, commerce, numérique

\section{AUTEURS}

GHISLAINE AZÉMARD

FMSH / Université Paris 8

\section{MATTHIEU QUINIOU}

FMSH / Université Paris 8 